\title{
Effects of Oils and Emulsifiers on the Skin Penetration of Stearyl Glycyrrhetinate in Oil-in-Water Emulsions
}

\author{
Osamu Sakata, ${ }^{a, b}$ Makiko Fujii, ${ }^{*}, a$ Naoya Koizumi, ${ }^{a}$ Masato Nakade, ${ }^{b}$ Koichi Kameyama, ${ }^{b}$ and \\ Yoshiteru Watanabe ${ }^{a}$ \\ ${ }^{a}$ Showa Pharmaceutical University; 3-3165 Higashi-Tamagawagakuen, Machida, Tokyo 194-8543, Japan: and \\ ${ }^{b}$ Research Laboratories, KOSÉ Corporation; 1-18-4 Azusawa, Itabashi-ku, Tokyo 174-0051, Japan. \\ Received August 29, 2013; accepted December 4, 2013
}

We investigated whether an emulsifier or an emulsified oil affects the skin penetration of stearyl glycyrrhetinate (SG) when it is applied in an oil-in-water $(\mathrm{O} / \mathrm{W})$-type emulsion under finite dose conditions in vitro. SG has a high molecular weight (MW: 723 ) and high lipophilicity (log $P$ : 15.6). Skin penetration of SG applied with $\mathrm{O} / \mathrm{W}$ emulsions was evaluated using 6 types of emulsifiers that are commonly used in cosmetics; however, no significant differences were observed between these emulsifiers. When applied with liquid paraffins in oil phase, SG skin penetration increased significantly as the molecular weight of the liquid paraffin decreased. The skin penetration of the fluorescent dye $1,1^{\prime}$-dioctadecyl-3,3,3',3'-tetramethylindocarbocyanine perchlorate (DiI; MW: 834, $\log P$ : 23.2) also increased with $\mathrm{O} / \mathrm{W}$-type emulsions containing liquid paraffins of lower molecular weights. These results indicate that use of $\mathrm{O} / \mathrm{W}$-type emulsions with an appropriate oil phase can improve SG skin penetration.

Key words skin penetration; stearyl glycyrrhetinate; finite dose; oil-in-water emulsion; liquid paraffin

Topical delivery of active ingredients occurs via partition and diffusion through the skin. Skin penetration depends on the logarithm of partition coefficient $(\log P)$ and the molecular weight of the compound. Compounds with molecular weights less than $500 \mathrm{Da}$ or with a $\log P$ of approximately 1 to 4 are most likely to be absorbed through the skin. ${ }^{1-3)}$ Thus, most of the drugs used in transdermal therapeutic systems are designed in accordance with these physicochemical parameters. ${ }^{4)}$ Many trials have investigated methods that might enhance the skin penetration of poorly absorbed compounds. For example, microemulsion applications have been used to enhance the skin delivery of polyphenols. ${ }^{5)}$ The effects of surfactants and lipids of microemulsions on skin permeation of octylmethoxycinnamate have also been investigated. $\left.{ }^{6}\right)$

Stearyl glycyrrhetinate (SG) (Fig. 1) is a derivative of glycyrrhetic acid, which is a well-known component of licorice and has anti-inflammatory and anti-viral properties. ${ }^{7,8)}$ It is a lipophilic compound that is frequently used in cosmetics such as emulsions and creams. The molecular weight of SG is $723 \mathrm{Da}$ and its $\log P$ is $\left.15.6 .^{9}\right)$ The properties of SG are outside the range of appropriate molecular weights and $\log P$. However, the target site of cosmetics is mainly the epidermis, and it is important to deliver the active ingredients to the epidermis. To date, there have been no reports on the skin penetration of SG. Thus, we examined whether emulsifiers or oils could improve SG skin penetration when applied as oil-in-water $(\mathrm{O} / \mathrm{W})$-type emulsions under finite dose conditions.

\section{MATERIALS AND METHODS}

Experimental Material SG was obtained from Maruzen Pharmaceuticals Co. (Hiroshima, Japan). 1,1'-Dioctadecyl3,3,3',3'-tetramethylindocarbocyanine perchlorate (DiI) was obtained from Sigma-Aldrich Japan Co. Liquid paraffins (LP) were obtained from Kaneda Co. (Tokyo, Japan), and their properties are shown in Table 1. All surfactants used (polyeth-

The authors declare no conflict of interest. ylene glycol-60 hydrogenated castor oil (HCO), hydrogenated soybean lecithin (HSL), polyethylene glycol 40EO monostearate (PMS), polysorbate 80 (PO80), sucrose monostearate (SMS), and sodium $N$-stearoyl-L-glutamate (SSG)) were of commercial cosmetic grade. Other reagents used were of analytical or HPLC grade.

Preparation of Oil-in-Water (O/W)-Type Emulsions Mixtures containing $0.5 \%(\mathrm{w} / \mathrm{v}) \mathrm{SG}, 5 \%(\mathrm{w} / \mathrm{v})$ of one of each of the emulsifiers (SMS, PO80, SSG, PMS, HSL, and HCO), and $20 \%(\mathrm{w} / \mathrm{v})$ LP-B were prepared in oil phase. Each emulsion was prepared using a high-pressure emulsifier (Microfluidizer, Powrex, Hyogo, Japan). Additionally, their particle size was made for approximately $200 \mathrm{~nm}$. The LP-A and LP-C emulsions were both prepared using the same procedure; mixtures containing $0.01 \%(\mathrm{w} / \mathrm{v}) \mathrm{DiI}, 5 \%(\mathrm{w} / \mathrm{v}) \mathrm{HCO}$, and $20 \%$ $(\mathrm{w} / \mathrm{v})$ LP-A or LP-C were prepared in oil phase. Their viscosities were $4.5 \mathrm{mPa} \cdot \mathrm{s}(\mathrm{LP}-\mathrm{A}), 4.4 \mathrm{mPa} \cdot \mathrm{s}(\mathrm{LP}-\mathrm{B})$, and $4.0 \mathrm{mPa} \cdot \mathrm{s}$ (LP-C), respectively. The DiI emulsion was prepared using an extruder with a 200-nm polycarbonate membrane filter (MiniExtruder, Avanti Polar Lipids, Alabama, U.S.A.). The oil suspension was prepared using the oil phase of $\mathrm{O} / \mathrm{W}$ emulsions. The composition ratio of oil suspensions was the same as that of the oil phase of the $\mathrm{O} / \mathrm{W}$ emulsions. The oil suspensions contained 2\%(w/v) SG, 20\% (w/v) HCO, and $80 \%(\mathrm{w} / \mathrm{v})$ of

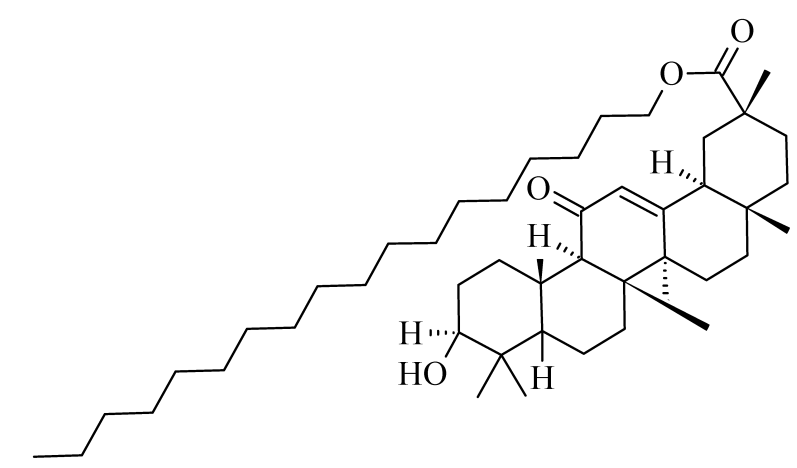

Fig. 1. Structure of Stearyl Glycyrrhetinate (SG) 
Table 1. Physical Properties of Liquid Paraffin (LP)-A, LP-B, and LP-C

\begin{tabular}{|c|c|c|c|c|}
\hline Liquid paraffin & MW & Surface tension $(\mathrm{mN} / \mathrm{m})$ & Viscosity (mPas) $32^{\circ} \mathrm{C}$ & SG solubility $(w t \%) 25^{\circ} \mathrm{C}$ \\
\hline LP-A & 245 & 27.6 & 3.0 & 0.55 \\
\hline LP-B & 330 & 29.1 & 13.0 & 0.41 \\
\hline LP-C & 495 & 30.7 & 78.2 & 0.23 \\
\hline
\end{tabular}

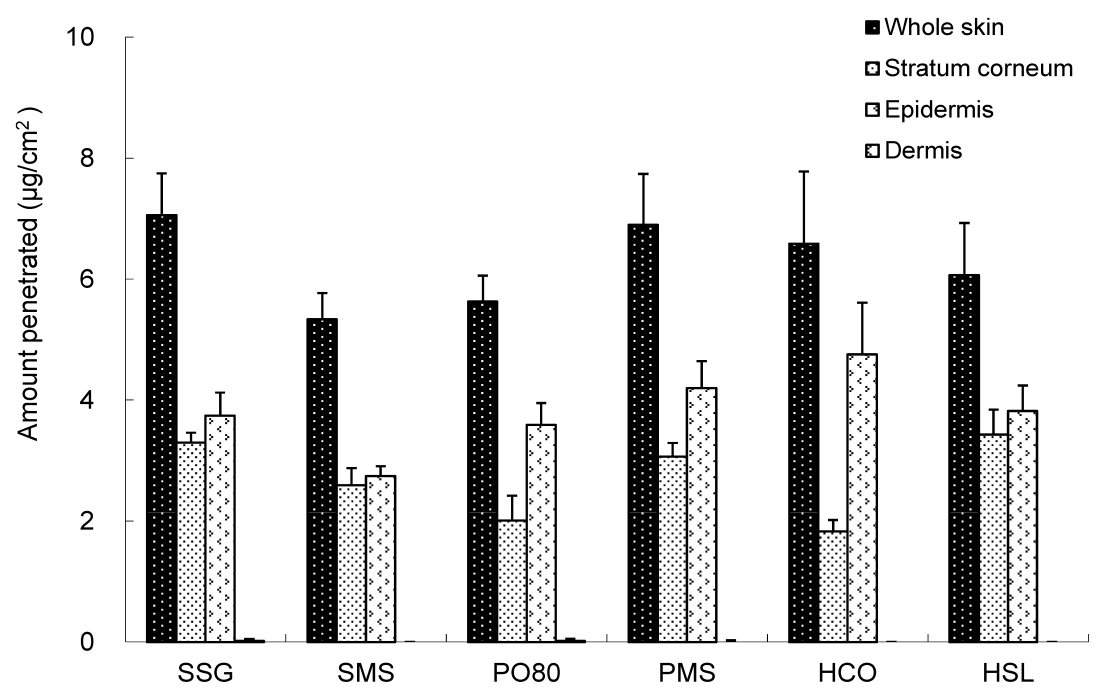

Fig. 2. Amount of Stearyl Glycyrrhetinate (SG) Penetration in the Skin $24 \mathrm{~h}$ after Application of Oil-in-Water (O/W) Preparations Emulsified with Various Surfactants

The amount of emulsion applied was $10 \mu \mathrm{L} / \mathrm{cm}^{2}$. Each column and bar represent the mean \pm S.D. of 4 experiments.

one of each of the LPs (LP-A, LP-B, and LP-C).

Skin Penetration Study Yucatan micropig (YMP) skin sets were purchased from Charles River, Japan, Inc. (Kanagawa, Japan). The skins were cut to $3 \times 3 \mathrm{~cm}^{2}$ and placed on a stainless steel tray filled with phosphate buffered saline (PBS) and $0.001 \%$ kanamycin, and incubated at $37^{\circ} \mathrm{C}$. SG emulsions $\left(10 \mu \mathrm{L} / \mathrm{cm}^{2}\right)$ or the oil phase containing SG $\left(2.5 \mu \mathrm{L} / \mathrm{cm}^{2}\right)$ were applied to the center of the skin covering an area of approximately $4 \mathrm{~cm}^{2}$. The skin was removed from the tray $24 \mathrm{~h}$ after application for further analysis.

Determination of SG Concentration in the Skin The skin surface was wiped with laboratory wipes (Kim-wipes; Nippon Paper Crecia, Tokyo, Japan) and stripped 10 times with adhesive tape (Scotch BH-18, 3M, Tokyo, Japan). The SG collected from the tape was considered to be "on the surface, ${ }^{, 10)}$ whereas that collected from the 3 rd to the 10th strips was considered to be from the "stratum corneum." The skin was then separated into the epidermis and dermis by using the heat separation method. SG that had penetrated the skin was extracted using $2 \mathrm{~mL}$ of methanol. The resulting solutions were then examined with HPLC to determine their SG concentrations. HPLC was performed using a Jasco instrument (LC-2000Plus series; Tokyo, Japan) equipped with a Mightysil RP-18GP C18 column $(5 \mu \mathrm{m}, 4.6 \mathrm{~mm} \times 150 \mathrm{~mm}$; Kanto Chemical, Tokyo, Japan) under the following conditions: mobile phase, methanol-ethanol $(75: 25, \mathrm{v} / \mathrm{v})$; column temperature, $40^{\circ} \mathrm{C}$; flow rate, $1 \mathrm{~mL} / \mathrm{min}$; and measurement wavelength, $254 \mathrm{~nm}$.

Determination of DiI Penetration into the Skin O/W emulsions containing DiI were applied to the skin by using the same procedure as that described for the SG skin penetration experiments, except the duration of the application periods differed. To better observe penetration of DiI into the stratum corneum, the examination time was adjusted to $2 \mathrm{~h}$. Following application of the emulsions, each skin sample was embedded in Frozen Section Compound (FSC22; Leica Microsystems, Tokyo, Japan) and frozen at $-80^{\circ} \mathrm{C}$. The frozen skin was cross-sectioned at a thickness of $14 \mu \mathrm{m}$ by using a Cryostat (CM3050S, Leica Microsystems) and observed by confocal laser scanning microscopy (CLSM, FLUOVIEW FV-1000; OLYMPUS, Tokyo, Japan) using a 543-nm He-Ne laser.

DiI suspension with LP-A was applied to the skin in two ways. One was a method whereby $10 \mu \mathrm{L}$ of water with $5 \%$ HCO was applied for 15 min before the application of DiI with liquid paraffin, and the other was a method whereby just DiI with liquid paraffin was applied. Two hours after application, the YMP skin was observed using the same procedure described above.

Statistical Analysis Statistical analysis was performed using JMP 9.03 software (SAS Institute Japan Inc., Tokyo, Japan). All data are presented as mean \pm S.D. Statistical analyses included ANOVA followed by Tukey-Kramer HSD test. A $p$ value of $<0.05$ was considered statically significant.

\section{RESULTS AND DISCUSSION}

We first examined whether emulsifiers affected SG skin penetration when applied in an $\mathrm{O} / \mathrm{W}$-type emulsion. $\mathrm{O} / \mathrm{W}$-type emulsions were prepared using 6 emulsifiers that are commonly used in cosmetics (an anionic ion surfactant SSG; nonionic surfactants SMS, PO80, PMS, and HCO; and an amphoteric ion surfactant HSL). The particle size of each emulsion was approximately $200 \mathrm{~nm}$, and each emulsion showed good stabil- 


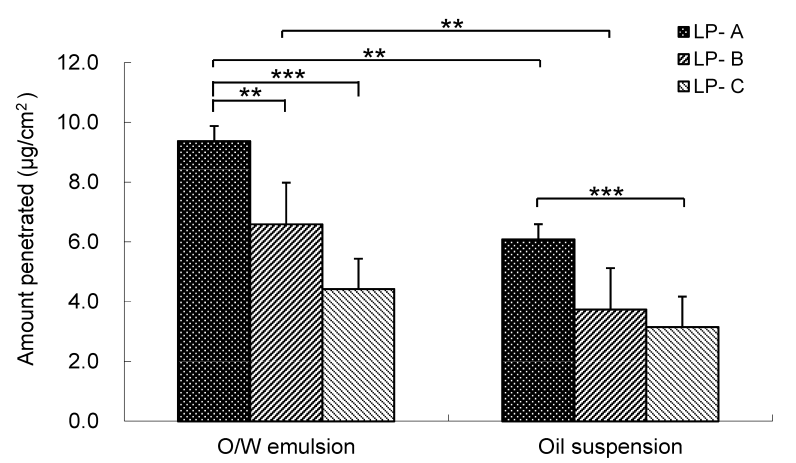

Fig. 3. Amount of Stearyl Glycyrrhetinate (SG) Penetration in the Skin $24 \mathrm{~h}$ after Application of an Oil-in-Water (O/W) Emulsion or a Corresponding Oil Phase Preparation, by Using 3 Types of Liquid Paraffin (LP)

Emulsions (SG, $0.5 \% \mathrm{w} / \mathrm{v}$ ) and oil phase preparations (SG, $2 \% \mathrm{w} / \mathrm{v}$ ) were applied at 10 and $2.5 \mu \mathrm{L} / \mathrm{cm}^{2}$, respectively $\left(50 \mu \mathrm{g} / \mathrm{cm}^{2} \mathrm{SG}\right)$. Each column and bar represent the mean \pm S.D. of 4 experiments. $* * * p<0.001, * * p<0.01$.

ity.

Figure 2 shows the amount of SG that penetrated the YMP skin. With HCO, SSG, and PMS, SG exhibited high levels of skin penetration. However, there was no significant difference between all formulations; the type of surfactant had little influence on SG skin penetration. The amount of SG that penetrated into the dermis was less than $1 \%$ for all emulsions, and most of the SG was detected in both the epidermis and the stratum corneum.

Next, we examined whether SG applied in the oil phase affected skin penetration by using the 3 types of LP with $\mathrm{HCO}$ as an emulsifier (Table 1). Figure 3 shows the amounts of $\mathrm{SG}$ that penetrated the skin from 3 different $\mathrm{O} / \mathrm{W}$ emulsions containing different types of liquid paraffin (LP-A, LP-B, and LP-C). The amount of SG skin penetration differed significantly between them (LP-A $>$ LP-B $>$ LP-C). The water contained in the $\mathrm{O} / \mathrm{W}$ emulsion evaporated on the skin, and the oil phase remained on the skin surface after phase inversion. Therefore, we also examined the skin penetration of the oil phase of the $\mathrm{O} / \mathrm{W}$ emulsion, and compared the emulsion with the oil phase. SG penetration into the skin was enhanced approximately 1.5 -fold with the $\mathrm{O} / \mathrm{W}$ emulsion, compared to the oil phase. We observed a significant difference between the $\mathrm{O} / \mathrm{W}$ emulsion and the oil phase (Fig. 3).

To clarify and visualize skin penetration, we used DiI, which has a molecular weight of $834 \mathrm{Da}$ and a $\log P$ of 23.2 . Figure 4 shows CLSM images of cross-sections of YMP skin after exposure to $\mathrm{O} / \mathrm{W}$ emulsions. DiI penetration into the stratum corneum differed significantly, with LP-A $>$ LP-C (Fig. 4). This phenomenon was also evident with regard to SG.

Furthermore, we sought to clarify one of the mechanisms by which skin penetration of $\mathrm{SG}$ with $\mathrm{O} / \mathrm{W}$ emulsions increases more than when the oil vehicle is used. The influence of skin hydration on the skin penetration of DiI by using an oil suspension of DiI and water was assessed. YMP skin was pretreated with water before the application of a DiI suspension. Figure 5 shows CLSM images of cross-sections of YMP skin after the application of a DiI suspension (a) and a DiI suspension with water pretreatment (b). The amount of DiI that penetrated the skin after pretreatment with water was more than that after application of a DiI suspension alone. This indicated that hydration of the skin increases the skin
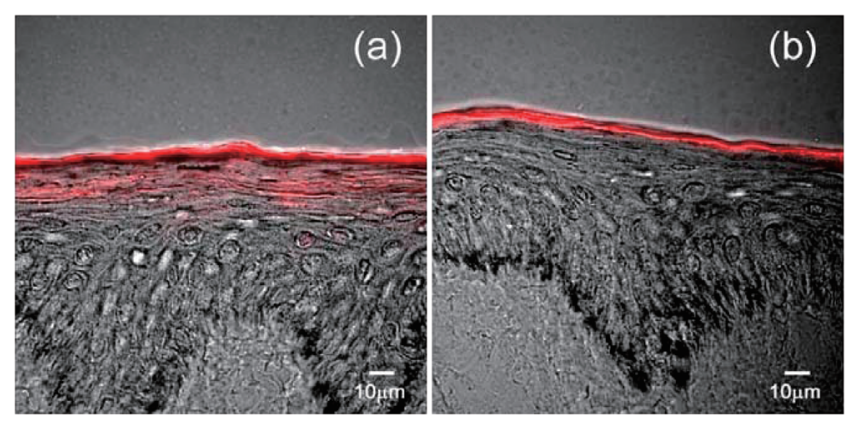

Fig. 4. Cross-Section of Yucatan Micropig Skin Observed by Confocal Laser Scanning Microscopy after Application of DiI as an Oil-in-Water (O/W)-Type Emulsion with (a) Liquid Paraffin (LP)-A, and (b) LP-C Bar indicates $10 \mu \mathrm{m}$.
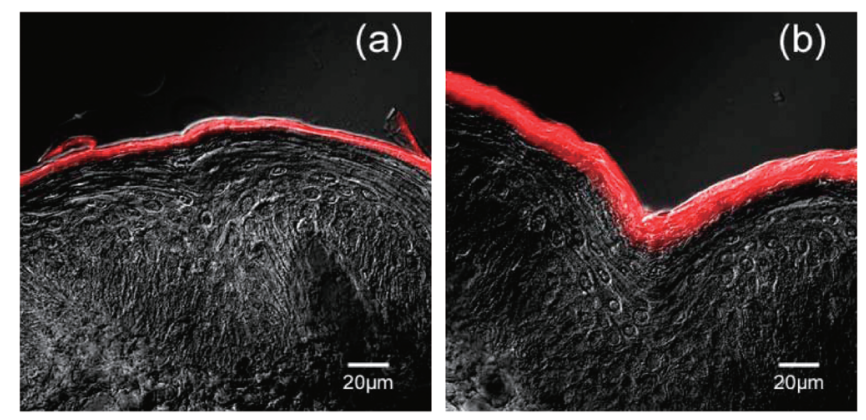

Fig. 5. Cross-Section of Yucatan Micropig Skin Observed by Confocal Laser Scanning Microscopy after Application of DiI with Liquid Paraffin (LP)-A, (a) without Pretreatment, and (b) with Pretreatment of Water before DiI Application

Bar indicates $20 \mu \mathrm{m}$.

\section{penetration of DiI.}

In our previous study, the skin penetration of SG was enhanced using oils with lower molecular weights and low surface tension as a vehicle. ${ }^{11)}$ The skin penetration order of $\mathrm{SG}$ in $\mathrm{O} / \mathrm{W}$ emulsions in the present study was similar to that observed in our previous study. Other explanations include that the skin penetration of compounds was affected by hydration of the stratum corneum. ${ }^{12}$ ) Our results indicate that the skin penetration of $\mathrm{SG}$ in $\mathrm{O} / \mathrm{W}$ emulsions is affected by both hydration and physical properties of the oil phase.

In the present study, we evaluated the skin penetration of $\mathrm{SG}$ and DiI applied in $\mathrm{O} / \mathrm{W}$ emulsions and under finite dose conditions. The amount of SG skin penetration was greater for the $\mathrm{O} / \mathrm{W}$-type emulsions than for the oil-phase preparations. Furthermore, skin penetration with the oil phase increased on using oils with lower molecular weights. We conclude that the extent of SG skin penetration can be enhanced using $\mathrm{O} / \mathrm{W}$ type emulsions containing LP with a low molecular weight.

\section{REFERENCES}

1) Bos JD, Meinardi MM. The 500 Dalton rule for the skin penetration of chemical compounds and drugs. Exp. Dermatol., 9, 165-169 (2000).

2) Zhang Q, Grice JE, Li P, Jepps OG, Wang GJ, Roberts MS. Skin solubility determines maximum transepidermal flux for similar size molecules. Pharm. Res., 26, 1974-1985 (2009).

3) Surber C, Wilhelm K, Maibach H. In vitro and in vivo percutaneous 
absorption of structurally related phenol and steroid analogs. Eur. J. Pharm. Biopharm., 39, 244-248 (1993).

4) Higo N. Recent trend of transdermal drug delivery system development. Yakugaku Zasshi, 127, 655-662 (2007).

5) Kitagawa S, Yoshii K, Morita SY, Teraoka R. Efficient topical delivery of chlorogenic acid by an oil-in-water microemulsion to protect skin against UV-induced damage. Chem. Pharm. Bull., 59, 793-796 (2011).

6) Montenegro L, Carbone C, Puglisi G. Vehicle effects on in vitro release and skin permeation of octylmethoxycinnamate from microemulsions. Int. J. Pharm., 405, 162-168 (2011).

7) Fiore C, Eisenhut M, Ragazzi E, Zanchin G, Armanini D. A history of the therapeutic use of liquorice in Europe. J. Ethnopharmacol., 99, 317-324 (2005)

8) Asl MN, Hosseinzadeh H. Review of pharmacological effects of Glycyrrhiza sp. and its bioactive compounds. Phytother. Res., 22,
709-724 (2008).

9) Cheng T, Zhao Y, Li X, Lin F, Xu Y, Zhang X, Li Y, Wang R, Lai L. Computation of octanol-water partition coefficients by guiding an additive model with knowledge. J. Chem. Inf. Model., 47, 2140-2148 (2007).

10) Shimoyama Y, Fujii M, Kanda Y, Mizoguchi A, Oda H, Koizumi $\mathrm{N}$, Watanabe Y. Effects of application method on skin penetration of carboxyfluorescein incorporated in liposomes. Chem. Pharm. Bull., 58, 429-431 (2010)

11) Sakata O, Fujii M, Koizumi N, Nakade M, Kameyama K, Watanabe Y. Effects of the physicochemical properties of oil vehicles on the skin penetration of poor skin-penetrable materials under finite dose conditions in vitro. Yakuzaigaku, 74, 84-92 (2014).

12) Hikima $T$, Maibach $H$. Skin penetration flux and lag-time of steroids across hydrated and dehydrated human skin in vitro. Biol. Pharm. Bull., 29, 2270-2273 (2006). 